\title{
A Numerical Algorithm on the Computation of the Stationary Distribution of a Discrete Time Homogenous Finite Markov Chain
}

\author{
Di Zhao, ${ }^{1}$ Hongyi Li, ${ }^{1}$ and Donglin $\mathrm{Su}^{2}$ \\ ${ }^{1}$ LMIB, School of Mathematics and System Science, Beihang University, Beijing 100191, China \\ ${ }^{2}$ Beihang Institute of EMC Technology, Beihang University, Beijing 100191, China
}

Correspondence should be addressed to Hongyi Li, hongyili_buaa@163.com

Received 8 January 2012; Revised 14 March 2012; Accepted 14 March 2012

Academic Editor: Zheng-Guang Wu

Copyright (C) 2012 Di Zhao et al. This is an open access article distributed under the Creative Commons Attribution License, which permits unrestricted use, distribution, and reproduction in any medium, provided the original work is properly cited.

The transition matrix, which characterizes a discrete time homogeneous Markov chain, is a stochastic matrix. A stochastic matrix is a special nonnegative matrix with each row summing up to 1 . In this paper, we focus on the computation of the stationary distribution of a transition matrix from the viewpoint of the Perron vector of a nonnegative matrix, based on which an algorithm for the stationary distribution is proposed. The algorithm can also be used to compute the Perron root and the corresponding Perron vector of any nonnegative irreducible matrix. Furthermore, a numerical example is given to demonstrate the validity of the algorithm.

\section{Introduction and Preliminaries}

Throughout this paper, the following notations and definitions are used. A matrix $A=\left(a_{i j}\right) \in$ $\mathbb{R}^{m \times n}$ is called nonnegative (positive), if all $a_{i j} \geq 0\left(a_{i j}>0\right)$, denoted by $A \geq 0(A>0)$. Similarly, a vector $x=\left(x_{1}, \ldots, x_{n}\right)^{T}$ is called nonnegative (positive) and denoted by $x \geq 0$ $(x>0)$, if all $x_{i} \geq 0\left(x_{i}>0\right)$. Let $B=\left(b_{i j}\right) \in \mathbb{R}^{m \times n}$, we denote $A \geq B(A>B)$, if $A-B \geq 0(>0)$, that is, $a_{i j} \geq b_{i j}\left(a_{i j}>b_{i j}\right)$ for all $i=1,2, \ldots, m, j=1,2, \ldots, n$.

For a square matrix $A \in \mathbb{R}^{n \times n}$ with eigenvalues $\lambda_{1}, \ldots, \lambda_{n}, \rho(A)=\max \left\{\left|\lambda_{j}\right|\right\}$ is called the spectral radius of $A$. If $A \geq 0$ is irreducible, there exists a unique eigenvector $x=\left(x_{1}, \ldots, x_{n}\right)^{T}>0$ such that $A x=\rho(A) x$ and $\|x\|_{1}=\left|x_{1}\right|+\cdots+\left|x_{n}\right|=1$. In this case, we say that $\rho(A)$ is the Perron root of $A$ and $x$ is the Perron vector [1].

We consider a discrete-time Markov chain $X=\left\{X^{(n)}: n=0,1, \ldots\right\}$ with a finite state space $\mathcal{S}=\left\{i_{1}, \ldots, i_{n}\right\}$. Among ergodic processes, homogeneous Markov chains with finite state space are particularly interesting examples. Such processes satisfy the Markov property, 
which states that their future behavior, conditional to the past and present, depends only on the present. Precisely, for all $t \in \mathbb{R}_{+}, h>0$, and for all sequences $0 \leq t_{1} \leq \cdots \leq t_{r}=t, i_{1}, \ldots, i_{r} \in$ $\mathcal{S}$ and $i_{j} \in \mathcal{S}$,

$$
P\left(X^{(t+h)}=i_{j} \mid X^{(t)}=i_{r}, X^{\left(t_{r}-1\right)}=i_{r-1}, \ldots, X^{\left(t_{1}\right)}=i_{1}\right)=P\left(X^{(h)}=i_{j} \mid X^{(0)}=i_{r}\right) .
$$

The behavior of such a process is characterized by an $n \times n$ matrix $M$ called the transition matrix [2].

Its stationary distribution $\pi$, which is also its asymptotic distribution, is a vector satisfying the following.

$$
\pi^{T} M=\pi^{T}, \quad \sum_{j=1}^{n} \pi_{j}=1,
$$

that is,

$$
\pi^{T} M=\pi^{T}, \quad \pi>0, \pi^{T} e=1,
$$

where $e$ is the column vector of all ones.

It has been established that it is possible to represent all possible uses of a software system as a Markov chain [3-5]. This model is called a Markov chain usage model. In a usage model, states of use (such as state "Document Loaded" in a model representing use of a word processing system) are represented by states in the Markov chain. Transitions between states of use (such as moving from state "Document Loaded" to "No Document Loaded" when the user closes a document in a word processing system) are represented by state transitions between the appropriate states in the Markov chain. Transitions between states of use have associated probabilities which represent the probability of making each transition. A usage model may be created based on information taken from functional specifications, usage specifications, and test objectives.

Considering the problem of software reliability, we represent a software system $S_{f}$ with $n$ states of use $\left\{s_{1}, \ldots, s_{n}\right\}$ by a homogeneous discrete Markov chain $\left\{X^{(n)}: n=0,1, \ldots\right\}$ (the corresponding transition matrix is $M$ ). We denote the initial state probability distribution $\pi^{(0)}=\left(\pi_{1}^{(0)}, \ldots, \pi_{n}^{(0)}\right)^{T}$, where $\pi_{i}^{(0)}=P\left(X^{(0)}=s_{i}\right)$. Then $\left(\pi^{(k)}\right)^{T}=\left(\pi^{(k-1)}\right)^{T} M$, where $\pi^{(k)}$ stands for the state probability distribution at time $k$. Let $\mu_{i}(i=1, \ldots, n)$ be the probability when the software fails at state $s_{i}$. The reliability of $S_{f}$ at time $k$ can be defined as $R^{(k)}=$ $1-\sum_{i=1}^{n} \mu_{i} \pi_{i}^{(k)}$. After a long time running, the state distribution of system $S_{f}$ will tend to the stationary distribution $\pi=\left(\pi_{1}, \ldots, \pi_{n}\right)^{T}$. Then, the terminating reliability $R=1-\sum_{i=1}^{n} \mu_{i} \pi_{i}$, with which we can evaluate the quality of a software system. By decreasing the $\mu_{i}$ of state $s_{i}$ with the largest probability $\pi_{i}$ in the stationary distribution $\pi$, we can also enhance the reliability of $S_{f}$ efficiently with limited resources.

A nonnegative matrix $A=\left(a_{i j}\right) \in \mathbb{R}^{n \times n}$ is called a row-stochastic matrix (or a stochastic matrix for short) if $\sum_{j=1}^{n} a_{i j}=1$ for all $i=1, \ldots, n$, that is, $A e=e$.

From the well-known Perron-Frobenius theorem, it can be easily deduced that the Perron root of a stochastic matrix $A$ equals 1.

Obviously, the transition matrix $M$ of a discrete-time homogeneous Markov chain is a stochastic matrix. From (1.3), we have $M^{T} \pi=\pi$. That is to say, the stationary distribution $\pi$ is also an eigenvector of $M^{T}$ associated to 1 . Since $M^{T}$ and $M$ have the same eigenvalues, $\pi$ is 
the Perron root of $M^{T}$, that is, the solution to $M^{T} \pi=\pi$. As for the computational aspects of $\pi$, many approaches have been presented (e.g., see [6-10]) based on the Gaussian elimination, direct projection and so on. In this paper, from the viewpoint of the Perron root which has not been discussed, we propose an algorithm for the stationary distribution $\pi$ considering that the computation of $\pi$ is equivalent to the computation of the Perron vector of $M$, which not only can compute the stationary distribution, but also could be used to compute the Perron root and the corresponding Perron vector of any nonnegative irreducible matrix (noting that the stationary distribution is the Perron vector of the transition matrix, which is a special nonnegative matrix).

This paper is organized as follows. In the next section, we propose some lemmas and preliminary results. In Section 3, we prove the convergent theorem and give some facts. In Section 4, we propose an algorithm for the stationary distribution together with a demonstrating numerical example.

\section{Some Lemmas}

In this section, we present some lemmas which will be used in the proof of the main results. The following facts can be found in [1, 11, 12].

Definition 2.1 (see [1]). Let $A \in \mathbb{R}^{n \times n}$ be a nonnegative matrix. If $A^{m}>0$ for some integer $m \geq 1$, one says that $A$ is primitive.

It is known that any primitive matrix must be irreducible [12]. We will use the following important facts which can be found in $[1,12]$.

Theorem A (see [1]). Let $A=A_{n \times n} \geq 0$, then $A$ is irreducible if and only if $(I+A)^{n-1}>0$, where $I$ is the unit matrix.

Theorem B (Perron-Frobenius (see [1])). Let $A=A_{n \times n} \geq 0$ be irreducible. Then,

(a) $\rho(A)>0$,

(b) $\rho(A)$ is an eigenvalue of $A$,

(c) There exists a vector $x>0$ such that $A x=\rho(A) x$,

(d) $\rho(A)$ is a simple eigen value of $A$.

This theorem guarantees the eigenspace of $\rho(A)$ is one-dimensional. That is, $A y=$ $\rho(A) y$ implies $y=k x$. And there exists an unique positive vector $x>0$ whose components sum to 1 such that $A x=\rho(A) x$. This $x$ is called the Perron vector [1].

For the Perron root of nonnegative matrices, many algorithms and bounds estimations have been proposed (see in $[13,14]$ ). In this paper, we will describe the Perron root by using the following Collatz-Wielandt functions [11, 12].

Definition 2.2 (see [11]). Let $A=\left(a_{i j}\right)_{n \times n}$ be nonnegative, define

$$
f_{A}(x)=\min \frac{(A x)_{j}}{x_{j}}, \quad g_{A}(x)=\max \frac{(A x)_{j}}{x_{j}}
$$

for any positive vector $x=\left(x_{1}, \ldots, x_{n}\right)^{T}>0$. 
$f_{A}(x)$ and $g_{A}(x)$ are both continuous at any $x>0$.

Lemma 2.3. Let $A=A_{n \times n}$ be nonnegative and irreducible. Then, for any $x>0, f_{A}(x)$ and $g_{A}(x)$ satisfy the following:

(1) $A x \geq f_{A}(x) x, A x \leq g_{A}(x) x$, and $f_{A}(x) \leq\|A\|_{1}$,

(2) $f_{A}(t x)=f_{A}(x), g_{A}(t x)=g_{A}(x)(t>0)$,

(3) Ax $>t x$ gives $f_{A}(x)>t$; Ax $<t x$ gives $g_{A}(x)<t$,

(4) If $B \geq 0$ is irreducible and $A B=B A$, let $y=B x$, then $f_{A}(y) \geq f_{A}(x)$ and $g_{A}(y) \leq g_{A}(x)$.

Proof. (1)-(3) are clearly true (see [10]). For (4), by $A x \geq f_{A}(x) x$, it follows that $A y=B(A x) \geq$ $B f_{A}(x) x=f_{A}(x) y$. This gives $f_{A}(y) \geq f_{A}(x)$. Similarly, $g_{A}(y) \leq g_{A}(x)$.

Lemma 2.4 (see [1]). If $B \geq 0$ is primitive ( $B^{m}>0$ for some $m \geq 1$ ), then

$$
\lim _{k \rightarrow \infty}\left[\rho(B)^{-1} B\right]^{k}=L>0, \quad B L=\rho(B) L
$$

\section{Main Results}

In this section, we will present the main results.

Theorem 3.1. If $A=A_{n \times n} \geq 0$ is irreducible and $B=B_{n \times n} \geq 0$ is primitive such that $A B=B A$. Let $x^{(0)}=\left(a_{1}, \ldots, a_{n}\right)^{T}>0$. Define for $k=1,2, \ldots$,

$$
y^{(k)}=B x^{(k-1)}, \quad x^{(k)}=\frac{y^{(k)}}{\left\|y^{(k)}\right\|_{1}} .
$$

Then,

(a) $\lim _{k \rightarrow \infty} x^{(k)}=x>0$, and $A x=\rho(A) x$ with $\|x\|_{1}=1$,

(b) $\lim _{k \rightarrow \infty} f_{A}\left(x^{(k)}\right)=\lim _{k \rightarrow \infty} g_{A}\left(x^{(k)}\right)=\rho(A)$,

(c) $f_{A}\left(x^{(0)}\right) \leq f_{A}\left(x^{(1)}\right) \leq \cdots \leq \rho(A) \leq \cdots \leq g_{A}\left(x^{(1)}\right) \leq g_{A}\left(x^{(0)}\right)$.

Proof. By (3.1), we can write $x^{(k)}=B^{k} x^{(0)} / b_{k}$ (for some $b_{k}>0$ ). This means for $k=1,2, \ldots$

$$
\left\|\frac{B^{k} x^{(0)}}{b_{k}}\right\|_{1}=\left\|x^{(k)}\right\|_{1}=1 .
$$

By Lemma 2.4,

$$
\lim _{k \rightarrow \infty}\left[\frac{B}{\rho(B)}\right]^{k}=L>0, \quad B L=\rho(B) L
$$


Equations (3.2) and (3.3) imply that

$$
\lim _{k \rightarrow \infty} \frac{b_{k}}{\rho(B)^{k}}=\left\|L x^{(0)}\right\|_{1^{\prime}} \quad \lim _{k \rightarrow \infty} x^{(k)}=\lim _{k \rightarrow \infty} \frac{B^{k} x^{(0)}}{b_{k}}=\frac{L x^{(0)}}{\left\|L x^{(0)}\right\|_{1}} .
$$

By putting $x=L x^{(0)} /\left\|L x^{(0)}\right\|_{1}$, it is clear that $x>0$ (with $\|x\|_{1}=1$ ) and

$$
B x=\frac{B L x^{(0)}}{\left\|L x^{(0)}\right\|_{1}}=\frac{\rho(B) L x^{(0)}}{\left\|L x^{(0)}\right\|_{1}}=\rho(B) x .
$$

Since $A B=B A$, we get $B(A x)=\rho(B) A x$. The Perron-Frobenius theorem (Theorem $\mathrm{B}$ ) guarantees that $\rho(B)$ is a simple eigenvalue of $B$. So, $B(A x)=\rho(B) A x$ gives that $A x=\lambda x(x>$ $0)$, which implies that $\lambda=\rho(A)$ and $A x=\rho(A) x$. On the other hand, by Definition 2.2, $A x=\rho(A) x(x>0)$ gives $f_{A}(x)=\rho(A)=g_{A}(x)$. By $\lim _{k \rightarrow \infty} x^{(k)}=x(x>0)$, we conclude that

$$
\lim _{k \rightarrow \infty} f_{A}\left(x^{(k)}\right)=f_{A}(x)=\rho(A), \quad \lim _{k \rightarrow \infty} g_{A}\left(x^{(k)}\right)=g_{A}(x)=\rho(A)
$$

By Lemma 2.3 and (3.1), we have for $k=1,2, \ldots$

$$
f_{A}\left(x^{(k-1)}\right) \leq f_{A}\left(x^{(k)}\right), \quad g_{A}\left(x^{(k)}\right) \leq g_{A}\left(x^{(k-1)}\right) .
$$

So, $\left\{f_{A}\left(x^{(k)}\right)\right\}$ and $\left\{g_{A}\left(x^{(k)}\right)\right\}$ are both monotonic convergent sequences. This proves (c), completing the proof.

Remark 3.2. From the proof, we know $A B x=\rho(A) \rho(B) x(x>0)$, and $\rho(A B)=\rho(A) \rho(B)$.

For an $n \times n$ irreducible matrix $A \geq 0$, since $(b I+A)^{n-1}>0(b>0), B=(b I+A)^{m}$ are primitive for $m=1,2, \ldots$. Clearly, $B A=A B$, we have the following.

Corollary 3.3. If $A=A_{n \times n} \geq 0$ is irreducible and let $B=(b I+A)^{m}$ (for fixed $m \geq 1$ and $b>0$ ). Let $x^{(0)}=\left(a_{1}, \ldots, a_{n}\right)^{T}>0$. For all $k=1,2, \ldots$, define

$$
y^{(k)}=B x^{(k)}, \quad x^{(k)}=\frac{y^{(k)}}{\left\|y^{(k)}\right\|_{1}} .
$$

Then,

(a) $\lim _{k \rightarrow \infty} x^{(k)}=x>0, A x=\rho(A) x,\left(\|x\|_{1}=1\right)$,

(b) $\lim _{k \rightarrow \infty} f_{A}\left(x^{(k)}\right)=\lim _{k \rightarrow \infty} g_{A}\left(x^{(k)}\right)=\rho(A)$,

(c) $f_{A}\left(x^{(0)}\right) \leq f_{A}\left(x^{(1)}\right) \leq \cdots \leq \rho(A) \leq \cdots \leq g_{A}\left(x^{(1)}\right) \leq g_{A}\left(x^{(0)}\right)$.

For a positive matrix $A>0$, all the matrices $B=A^{m}(m=1,2, \ldots)$ are primitive. The following is obvious. 
Corollary 3.4. If $A=A_{n \times n}>0$ and $B=A^{m}$ (for $\left.m \geq 1\right)$. Let $x^{(0)}=\left(a_{1}, \ldots, a_{n}\right)^{T}$ be a positive vector. Define

$$
y^{(k)}=B x^{(k)}, \quad x^{(k)}=\frac{y^{(k)}}{\left\|y^{(k)}\right\|_{1}} \quad \forall k=1,2, \ldots
$$

Then,

(a) $\lim _{k \rightarrow \infty} x^{(k)}=x>0, A x=\rho(A) x,\left(\|x\|_{1}=1\right)$,

(b) $\lim _{k \rightarrow \infty} f_{A}\left(x^{(k)}\right)=\lim _{k \rightarrow \infty} g_{A}\left(x^{(k)}\right)=\rho(A)$,

(c) $f_{A}\left(x^{(0)}\right) \leq f_{A}\left(x^{(1)}\right) \leq \cdots \leq \rho(A) \leq \cdots \leq g_{A}\left(x^{(1)}\right) \leq g_{A}\left(x^{(0)}\right)$.

By (3.1), let $\lambda^{(k)}=(1 / 2)\left(f_{A}\left(x^{(k)}\right)+g_{A}\left(x^{(k)}\right)\right)$ for $(k=1,2, \ldots)$, one has the following.

Corollary 3.5. If $g_{A}\left(x^{(k)}\right)-f_{A}\left(x^{(k)}\right)<\varepsilon(\varepsilon>0)$, then $\left|\rho(A)-\lambda^{(k)}\right|<(1 / 2) \varepsilon$.

Proof. From Theorem 3.1, it follows that

$$
\begin{aligned}
\left|\rho(A)-\lambda^{(k)}\right| & \leq \frac{1}{2}\left|\rho(A)-f_{A}\left(x^{(k)}\right)\right|+\frac{1}{2}\left|\rho(A)-g_{A}\left(x^{(k)}\right)\right| \\
& =\frac{1}{2}\left(\rho(A)-f_{A}\left(x^{(k)}\right)\right)+\frac{1}{2}\left(g_{A}\left(x^{(k)}\right)-\rho(A)\right) \\
& =\frac{1}{2}\left(g_{A}\left(x^{(k)}\right)-f_{A}\left(x^{(k)}\right)\right)<\frac{1}{2} \varepsilon .
\end{aligned}
$$

If $A \geq 0$ is irreducible, it is obvious that $B=b I+A(b>0)$ is primitive, and $\rho(A)=$ $\rho(B)-b$. So, we have the following.

Corollary 3.6. If $A \geq 0$ is irreducible, for any $b>0$, let $B=b I+A$. Using the sequences $\left\{f_{B}\left(x^{(k)}\right)\right\}$ and $\left\{g_{B}\left(x^{(k)}\right)\right\}$. Then,

$$
\begin{gathered}
\lim _{k \rightarrow \infty} f_{B}\left(x^{(k)}\right)=\lim _{k \rightarrow \infty} g_{B}\left(x^{(k)}\right)=\rho(B) ; \quad \lim _{k \rightarrow \infty} x^{(k)}=x>0, \\
\left(\|x\|_{1}=1\right) ; \quad \rho(A)=\rho(B)-b .
\end{gathered}
$$

\section{An Algorithm and a Numerical Example}

In this section, we propose a numerical algorithm to compute the stationary distribution of a discrete time homogeneous finite Markov chain.

Algorithm 4.1 (to compute the stationary distribution $\pi$ ). Step 1. Giving a transition matrix $M$ of a discrete time homogeneous finite Markov chain, a calculation precision $\varepsilon>0$. Choosing parameters: a positive real number $b>0$ and an integer $m$. Setting the initial iterative vector $\pi^{(0)}=(1,1, \ldots, 1)^{T}, B=\left(b I+M^{T}\right)^{m}, k=1$. 
Table 1: Iteration results of Example 4.3 by Algorithm 4.1.

\begin{tabular}{|c|c|c|c|c|}
\hline$k$ & $y^{(k)}$ & $\pi^{(k)}$ & $f_{M^{T}}\left(\pi^{(k)}\right)$ & $g_{M^{T}}\left(\pi^{(k)}\right)$ \\
\hline \multirow{6}{*}{1} & 9.8480005 & 0.2051667 & \multirow{6}{*}{0.8266823} & \multirow{6}{*}{1.0792825} \\
\hline & 4.2060003 & 0.0876250 & & \\
\hline & 8.2880001 & 0.1726667 & & \\
\hline & 8.1380005 & 0.1695417 & & \\
\hline & 6.8360000 & 0.1424167 & & \\
\hline & 10.6840000 & 0.2225833 & & \\
\hline \multirow{6}{*}{2} & 1.7046144 & 0.2130768 & \multirow{6}{*}{0.9024122} & \multirow{6}{*}{1.0330924} \\
\hline & 0.6064956 & 0.0758119 & & \\
\hline & 1.4543651 & 0.1817956 & & \\
\hline & 1.4877499 & 0.1859687 & & \\
\hline & 0.8997051 & 0.1124631 & & \\
\hline & 1.8470705 & 0.2308838 & & \\
\hline \multirow{6}{*}{3} & 1.7026380 & 0.2128298 & \multirow{6}{*}{0.9654347} & \multirow{6}{*}{1.0107353} \\
\hline & 0.6010615 & 0.0751327 & & \\
\hline & 1.4877805 & 0.1859726 & & \\
\hline & 1.5420293 & 0.1927537 & & \\
\hline & 0.8042196 & 0.1005275 & & \\
\hline & 1.8622712 & 0.2327839 & & \\
\hline \multirow{6}{*}{4} & 1.6968167 & 0.2121021 & \multirow{6}{*}{0.9906667} & \multirow{6}{*}{1.0030047} \\
\hline & 0.6042080 & 0.0755260 & & \\
\hline & 1.4985833 & 0.1873229 & & \\
\hline & 1.4985833 & 0.1949336 & & \\
\hline & 0.7754812 & 0.0969351 & & \\
\hline & 1.8654419 & 0.2331802 & & \\
\hline \multirow{6}{*}{5} & 1.6940787 & 0.2117598 & \multirow{6}{*}{0.9979988} & \multirow{6}{*}{1.0010511} \\
\hline & 0.6060677 & 0.0757585 & & \\
\hline & 1.5014149 & 0.1876768 & & \\
\hline & 1.5642191 & 0.1955274 & & \\
\hline & 0.7683222 & 0.0960403 & & \\
\hline & 1.8658978 & 0.2332372 & & \\
\hline \multirow{6}{*}{6} & 1.6931415 & 0.2116427 & \multirow{6}{*}{0.9997041} & \multirow{6}{*}{1.0003293} \\
\hline & 0.6067572 & 0.0758447 & & \\
\hline & 1.5020157 & 0.1877520 & & \\
\hline & 1.5653062 & 0.1956633 & & \\
\hline & 0.7668935 & 0.0958617 & & \\
\hline & 1.8658856 & 0.2332357 & & \\
\hline \multirow{6}{*}{7} & 1.6928755 & 0.2116094 & \multirow{6}{*}{0.9999617} & \multirow{6}{*}{1.0000869} \\
\hline & 0.6069643 & 0.0758705 & & \\
\hline & 1.5021036 & 0.1877629 & & \\
\hline & 1.5654967 & 0.1956871 & & \\
\hline & 0.7667158 & 0.0958395 & & \\
\hline & 1.8658446 & 0.2332305 & & \\
\hline \multirow{4}{*}{8} & 1.6928117 & 0.2116015 & \multirow{4}{*}{0.9999923} & \\
\hline & 0.6070167 & 0.0758771 & & \\
\hline & 1.5021019 & 0.1877628 & & 1.0000242 \\
\hline & 1.5655103 & 0.1956888 & & $1.0000 \angle 4 Z$ \\
\hline
\end{tabular}


Table 1: Continued.

\begin{tabular}{|c|c|c|c|c|}
\hline$k$ & $y^{(k)}$ & $\pi^{(k)}$ & $f_{M^{T}}\left(\pi^{(k)}\right)$ & $g_{M^{T}}\left(\pi^{(k)}\right)$ \\
\hline & 0.7667347 & 0.0958418 & & \\
\hline & 1.8658243 & 0.2332281 & & \\
\hline \multirow{6}{*}{9} & 1.6927999 & 0.2116000 & \multirow{6}{*}{0.9999974} & \multirow{6}{*}{1.0000122} \\
\hline & 0.6070276 & 0.0758785 & & \\
\hline & 1.5020945 & 0.1877618 & & \\
\hline & 1.5655029 & 0.1956879 & & \\
\hline & 0.7667580 & 0.0958448 & & \\
\hline & 1.8658174 & 0.2332272 & & \\
\hline \multirow{6}{*}{10} & 1.6927987 & 0.2115998 & \multirow{6}{*}{0.9999989} & \multirow{6}{*}{1.0000043} \\
\hline & 0.6070291 & 0.0758786 & & \\
\hline & 1.5020909 & 0.1877613 & & \\
\hline & 1.5654980 & 0.1956872 & & \\
\hline & 0.7667685 & 0.0958460 & & \\
\hline & 1.8658154 & 0.2332269 & & \\
\hline \multirow{6}{*}{11} & 1.6927989 & 0.2115999 & \multirow{6}{*}{0.9999996} & \multirow{6}{*}{1.0000013} \\
\hline & 0.6070290 & 0.0758786 & & \\
\hline & 1.5020894 & 0.1877612 & & \\
\hline & 1.5654960 & 0.1956870 & & \\
\hline & 0.7667719 & 0.0958465 & & \\
\hline & 1.8658147 & 0.2332269 & & \\
\hline \multirow{6}{*}{12} & 1.6927993 & 0.2115999 & \multirow{6}{*}{0.9999998} & \multirow{6}{*}{1.0000004} \\
\hline & 0.6070290 & 0.0758786 & & \\
\hline & 1.5020891 & 0.1877611 & & \\
\hline & 1.5654955 & 0.1956869 & & \\
\hline & 0.7667729 & 0.0958466 & & \\
\hline & 1.8658148 & 0.2332268 & & \\
\hline
\end{tabular}

Step 2. Computing $\pi^{(k)}$ from $\pi^{(k-1)}$ :

$$
y^{(k)}=B \pi^{(k-1)}, \quad \pi^{(k)}=\frac{y^{(k)}}{\left\|y^{(k)}\right\|_{1}} \quad(\forall k=1,2, \ldots)
$$

Step 3. Compute $f_{M^{T}}\left(\pi^{(k)}\right)$ and $g_{M^{T}}\left(\pi^{(k)}\right)$ :

$$
f_{M^{T}}\left(\pi^{(k)}\right)=\min _{1 \leq i \leq n} \frac{\left(M^{T} \pi^{(k)}\right)_{i}}{\pi_{i}^{(k)}}, \quad g_{M^{T}}\left(\pi^{(k)}\right)=\max _{1 \leq i \leq n} \frac{\left(M^{T} \pi^{(k)}\right)_{i}}{\pi_{i}^{(k)}} .
$$
to Step 2.

Step 4. If $g_{M^{T}}\left(\pi^{(k)}\right)-f_{M^{T}}\left(\pi^{(k)}\right)<\varepsilon$, go to Step 5. Otherwise setting $k:=k+1$, go back

Step 5. Let $\lambda=(1 / 2)\left(f_{M^{T}}\left(\pi^{(k)}\right)+g_{M^{T}}\left(\pi^{(k)}\right)\right)$. Then $\lambda$ is the approximation of the Perron root of $M^{T}$, and the corresponding $\pi^{(k)}$ is the approximation of the stationary distribution of M.

Remark 4.2. From Theorem 3.1, the convergence of Algorithm 4.1 is obvious.

We next give a numerical example. 
Example 4.3. For a given finite Markov Chain, with the corresponding transition matrix as the following:

$$
M=\left[\begin{array}{cccccc}
0.2 & 0 & 0.3 & 0.1 & 0 & 0.4 \\
0 & 0.1 & 0.2 & 0 & 0.5 & 0.2 \\
0.5 & 0 & 0 & 0.1 & 0 & 0.4 \\
0 & 0.3 & 0.2 & 0.2 & 0.1 & 0.2 \\
0.3 & 0.1 & 0 & 0 & 0.4 & 0.2 \\
0.2 & 0 & 0.3 & 0.5 & 0 & 0
\end{array}\right]
$$

finding its approximating stationary distribution with calculation precision $\varepsilon=10^{-6}$.

By choosing the initial iterative vector $x^{(0)}=(1,1,1,1,1,1)^{T}$, parameters $m=3, b=1$, that is, $B=\left(I+M^{T}\right)^{3}$, and applying Algorithm 4.1, the approximating Perron root and Perron vector are obtained after 12 iterations:

$$
\begin{gathered}
\pi^{(12)}=(0.2115999,0.0758786,0.18776110,0.1956869,0.0958466,0.2332268)^{T}, \\
\lambda^{(12)}=1.0000001 .
\end{gathered}
$$

The iteration results are listed in Table 1.

\section{Acknowledgment}

The project was supported by the National Natural Science Foundation of China (Grant no. 60831001).

\section{References}

[1] R. A. Horn and C. R. Johnson, Matrix Analysis, Cambridge University Press, Cambridge, UK, 1985.

[2] P. Regnault, "Estimation using plug-in of the stationary distribution and Shannon entropy of continuous time Markov processes," Journal of Statistical Planning and Inference, vol. 141, no. 8, pp. 2711-2725, 2011.

[3] J. H. Poore, H. D. Mills, and D. Mutchler, "Planning and certifying software system reliability," IEEE Software, pp. 88-99, 1993.

[4] J. H. Poore and C. J. Trmmell, "Application of statistical science to testing and evaluating software intensive systems," in Statostics, Testing and Defense Acquisition, National Academy Press, Washington, DC, USA, 1998.

[5] J. A. Whittaker and M. G. Thomason, "Markov chain model for statistical software testing," IEEE Transactions on Software Engineering, vol. 20, no. 10, pp. 812-824, 1994.

[6] M. Benzi, "A direct projection method for Markov chains," Linear Algebra and Its Applications, vol. 386, pp. 27-49, 2004.

[7] D. P. Heyman and A. Reeves, "Numerical solution of linear equations arising in Markov chain models," ORSA Journal on Computing, vol. 1, pp. 52-60, 1989.

[8] I. Marek, "Iterative aggregation/disaggregation methods for computing some characteristics of Markov chains. II. Fast convergence," Applied Numerical Mathematics, vol. 45, no. 1, pp. 11-28, 2003.

[9] M. Neumann and J. Xu, "On the stability of the computation of the stationary probabilities of Markov chains using Perron complements," Numerical Linear Algebra with Applications, vol. 10, no. 7, pp. 603618, 2003.

[10] C. C. Paige, G. P. H. Styan, and P. G. Wachter, "Computation of the stationary distribution of a Markov chain," Journal of Statistical Computation and Simulation, vol. 4, pp. 173-186, 1975. 
[11] H. Minc, Nonnegative Matrices, Wiley-Interscience Series in Discrete Mathematics and Optimization, John Wiley \& Sons, New York, NY, USA, 1988.

[12] R. S. Varga, Matrix Iterative Analysis, vol. 27 of Springer Series in Computational Mathematics, Springer, Berlin, Germany, 2000.

[13] F. Duan and K. Zhang, "An algorithm of diagonal transformation for Perron root of nonnegative irreducible matrices," Applied Mathematics and Computation, vol. 175, no. 1, pp. 762-772, 2006.

[14] H. Y. Li, D. Zhao, and F. Dai, "On the spectral radius of a nonnegative centrosymmetric matrix," Applied Mathematics and Computation, vol. 218, pp. 4962-4966, 2012. 


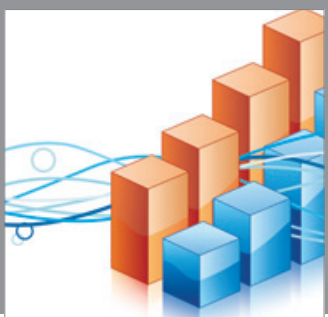

Advances in

Operations Research

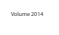

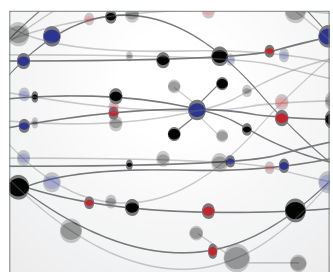

\section{The Scientific} World Journal
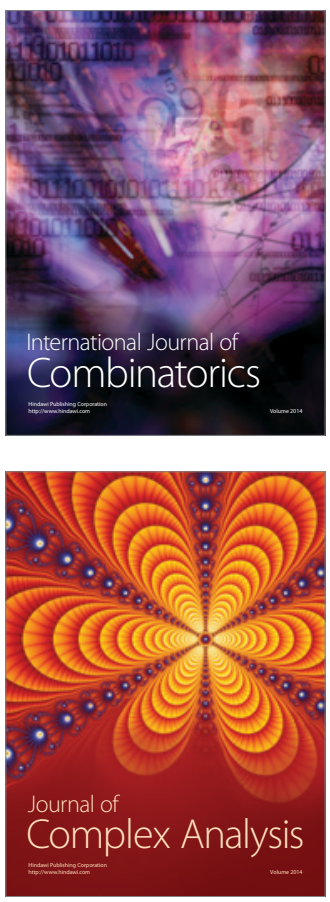

International Journal of

Mathematics and

Mathematical

Sciences
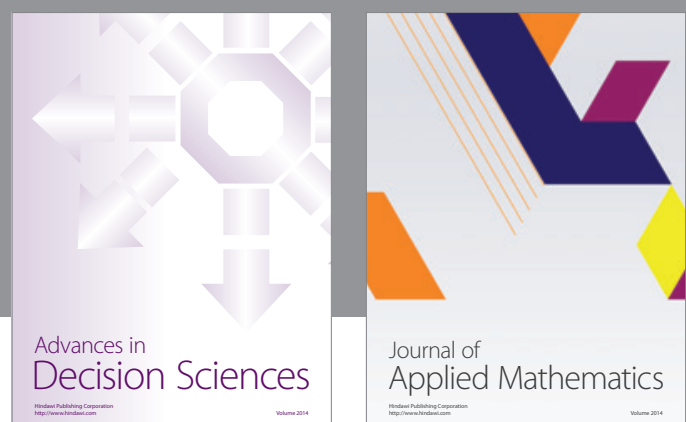

Journal of

Applied Mathematics
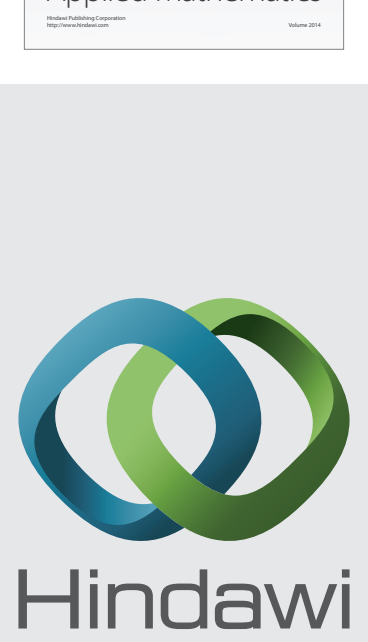

Submit your manuscripts at http://www.hindawi.com
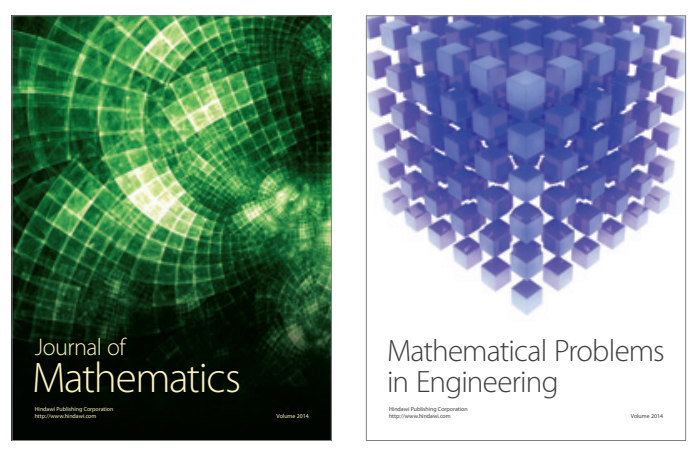

Mathematical Problems in Engineering
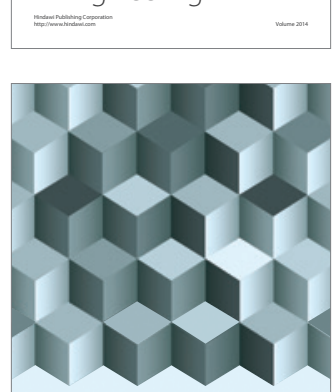

Journal of

Function Spaces
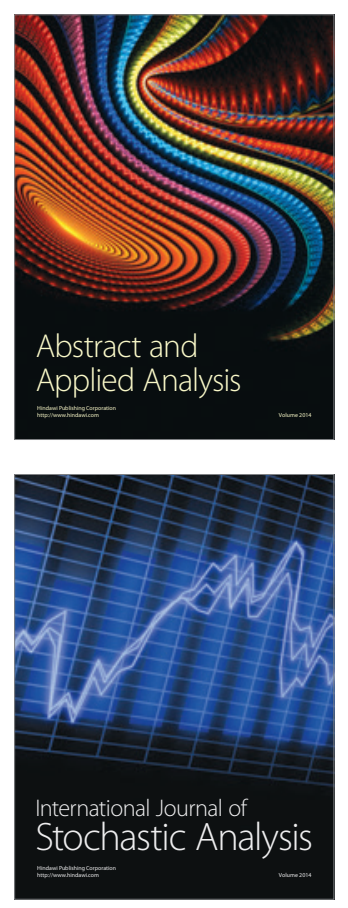

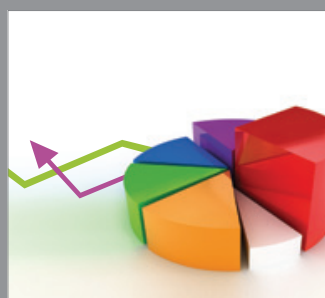

ournal of

Probability and Statistics

Promensencen
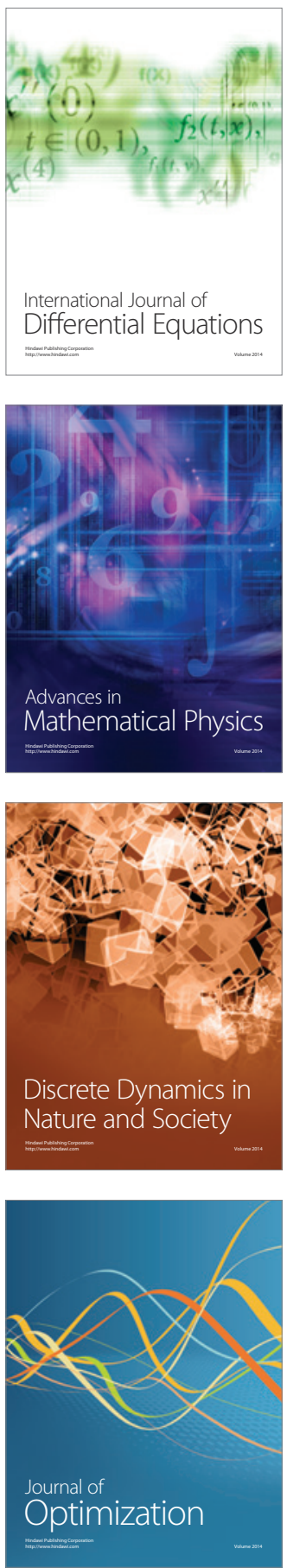